\title{
Zwitterion-coated Colloidal Magnetic Nanoparticle Clusters for Reduced Nonspecific Adsorption of Biomolecules
}

Gyudong Lee, ${ }^{1}$ Jaejung Song, ${ }^{2}$ Hyunsoo Han, ${ }^{3}$ Donghoon Kwon, ${ }^{3}$ Joonhyuck Park, ${ }^{1}$ Sangmin Jeon, ${ }^{3}$ Sanghwa Jeong ${ }^{1,4 *}$ and Sungjee Kim ${ }^{1,2 *}$

1Department of Chemistry, Pohang University of Science \& Technology (POSTECH), 77 Cheongam-Ro,
Nam-Gu, Pohang, Gyeongbuk, 37673, South Korea
${ }^{2}$ School of Interdisciplinary Bioscience and Bioengineering, POSTECH, 37673, South Korea
${ }^{3}$ Department of Chemical Engineering, POSTECH, 37673, South Korea
${ }^{4}$ School of Biomedical Convergence Engineering, Pusan National University, Yangsan, 50612, South Korea

\section{Table of Contents}

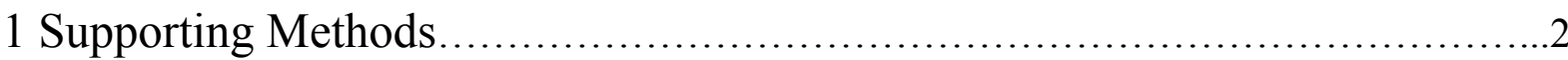

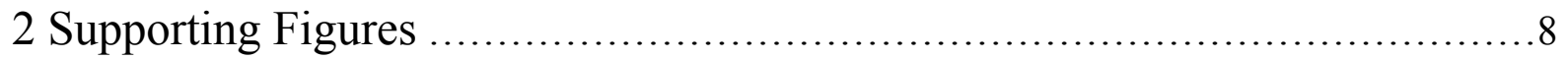

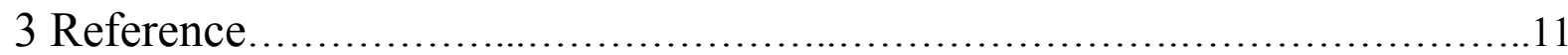




\section{Supporting Methods}

\section{Material and characterization}

Iron chloride hexahydrate, sodium citrate tribasic dihydrate, urea, polyacrylamide $\left(\mathrm{M}_{\mathrm{w}}\right.$ 5,000,000-6,000,000), 1,3-propanesultone, tetraethylorthosilicate, $\quad(+)$-Biotin $\quad N$ hydroxysuccinimide ester (NHS-biotin), Streptavidin-Agarose bead, 1,1-carbonyldiimidazole (CDI), $\quad N$-(3-Dimethylaminopropyl)- $N$ '-ethylcarbodiimide hydrochloride $\quad$ (EDC), $\quad N$ Hydroxysulfosuccinimide sodium salt (sulfo-NHS), ( \pm )- $\alpha$-Lipoic acid, $N, N$ Dimethylethylenediamine (DMEN), streptavidin, glutaraldehyde solution were purchased from Aldrich. ( $N, N$-dimethyl-3-aminopropyl)trimethoxysilane and (3-aminopropyl)triethoxysilane (APTES) were purchased from Tokyo chemical industry. $1 \mathrm{M}$ phosphate buffered saline (PBS) were purchased from Hyclone. 2-( $N$-morpholino)ethanesulfonic acid (MES) was purchased from Acros. Fluoresacmine was obtained from Alfa Aesar. Goat anti-Salmonella CSA-1 Molecular Grade Antibody was purchased from KPL, Inc. Other reagents were purchased from Samchun chemical. Water was triply distilled using a Millipore filtration system.

Hydrodynamic size and zeta potential were obtained using Malvern Zetasizer Nano S and Zetasizer Nano Z, respectively. Photoluminescence (PL) spectra were acquired using a HORIBA FluoroLog instrument. Nuclear magnetic resonance (NMR) spectrum was obtained with Bruker Advance III Ascend 600. Transmission electron microscopy (TEM) and scanning electron microscopy (SEM) images were recorded with a JEOL JEM-2100 and JSM-7401F, respectively.

\section{Synthesis of magnetic nanoparticles clusters (MNCs)}

MNCs were synthesized following a hydrothermal method described in the literature. ${ }^{[1]}$ First, 3 mmol of $\mathrm{FeCl}_{3} \cdot 6 \mathrm{H}_{2} \mathrm{O}, 6 \mathrm{mmol}$ of sodium citrate tribasic dihydrate, $9 \mathrm{mmol}$ of urea, and $60 \mathrm{nmol}$ of polyacrylamide were dissolved in $60 \mathrm{~mL}$ of deionized water. The solution was heated to 200 ${ }^{\circ} \mathrm{C}$ in a Teflon-lined autoclave and maintained at that temperature for $19 \mathrm{~h}$. The autoclave was 
cooled to room temperature, then MNCs were separated using a magnet and washed several times with deionized water and ethanol. The resulting MNCs were dispersed in deionized water for further use. (About 36 pmol of MNC were synthesized.)

\section{Synthesis of 3-(Dimethyl(3-(trimethoxysilyl)propyl)-ammonio)propane-1-sulfonate}

3-(Dimethyl(3-(trimethoxysilyl)propyl)-ammonio)propane-1-sulfonate (zwitterionic siloxane, ZW) was prepared using a method from the literature. ${ }^{[2]}$ First, $7 \mathrm{mmol}$ of ( $N, N$-dimethyl-3aminopropyl)trimethoxysilane were dissolved in $7.4 \mathrm{~mL}$ of acetone, then $7 \mathrm{mmol}$ of 1,3propanesultone were added to the solution. The reaction mixture was stirred for $6 \mathrm{~h}$ under $\mathrm{N}_{2}$ atmosphere. The white solid was collected by vacuum filtration and washed with excess acetone. The result solid was dried under vacuum at $30^{\circ} \mathrm{C}$.

\section{Silanization of MNC}

MNCs (1 pmol) were dispersed in $8 \mathrm{~mL}$ deionized water and $40 \mathrm{~mL}$ ethanol. Then $1 \mathrm{~mL}$ of $\mathrm{NH}_{3}$ solution (28-30 wt $\%$ ) and $40 \mu \mathrm{L}$ of tetraethylorthosilicate were added to the MNC solution, and it was vortexed vigorously for $3 \mathrm{~h}$. The resulting silica-coated MNCs (SMNCs) were collected using a magnet, then washed with deionized water ethanol. For further surface modification, SMNCs were dispersed in deionized water.

\section{Surface modification of SMNC with zwitterionic siloxane and (3-aminopropyl) triethoxysilane}

SMNCs $(0.1 \mathrm{pmol})$ were dispersed in $1.95 \mathrm{~mL}$ deionized water. The desired ratio of $\mathrm{ZW}$ and (3-aminopropyl)triethoxysilane (APTES) was added to the SMNC solution while the total amount of surface ligands was maintained at $100 \mu \mathrm{mol}$. Then $50 \mu \mathrm{L}$ of $\mathrm{NH}_{3}$ solution was added. The reaction mixture was vortexed for $6 \mathrm{~h}$ at room temperature. The products were SMNCs 
decorated with zwitterion groups and amine groups (SMNC-ZW/Am); they were separated using a magnet, then washed several times with deionized water.

\section{Biotinylation of SMNC-ZW/Am}

As-prepared SMNC-ZW-3 (0.1 pmol, ZW:APTES = 95:5) was dispersed in 0.01 M of PBS buffer ( $\mathrm{pH}$ 7.4). Then $10 \mu \mathrm{L}$ of NHS-biotin solution ( $2 \mathrm{mg} / \mathrm{mL}$, in DMSO) was added to SMNC3 solution. (The volume of SMNC-ZW-3 solution was $<100 \mu \mathrm{L}$.) The mixture was vortexed for $2 \mathrm{~h}$, then the resulting bionylated SMNC-ZW-3 (SMNC-ZW-3-b) was separated using a magnet, and washed with deionized water.

To characterize of biotinylation, streptavidin-agarose bead were mixed with biotinylated SMNC-ZW-3 and unconjugated SMNC-ZW-3 with free biotin for $30 \mathrm{~min}$. Then excess SMNCZW-3 was washed off using deionized water in a filter funnel. (Ratio of SMNC-ZW/Am : streptavidin-agarose bead $\left.=10^{7}: 1\right)$

\section{Synthesis of zwitterion decorated lipoic acid}

Zwitterion-decorated lipoic acid (ZA) were synthesized using tertiary amine decorated lipoic acid (TA) and 1,3-propanesultone, as described previously ${ }^{[3]}$. To synthesize TA, $20 \mathrm{mmol}$ of lipoic acid (LA) and $26 \mathrm{mmol}$ of 1,1-carbonyldiimidazole were dissolved in $30 \mathrm{~mL}$ of anhydrous chloroform and stirred under $\mathrm{N}_{2}$ atmosphere for $20 \mathrm{~min}$. Then the reaction flask was placed in an ice bath; $100 \mathrm{mmol}$ of $N, N$-Dimethylethylenediamine was added to reaction mixture and stirred for $12 \mathrm{~h}$ under $\mathrm{N}_{2}$ atmosphere. An extraction was conducted using $100 \mathrm{mM}$ $\mathrm{NaCl}$ solution and $10 \mathrm{mM} \mathrm{NaOH}$ solution to remove excess reagent and byproduct. The result TA solution was dried with anhydrous magnesium sulfate.

To synthesize ZA, 1,3-propanesultone was added to TA solution $\left(60 \mathrm{~mL} \mathrm{CHCl}_{3}\right)$ and the solution was stirred for $24 \mathrm{~h}$. A a yellow solid precipitated; it was was collected by vacuum filtration and washed with anhydrous chloroform. 


\section{Synthesis of streptavidin-conjugated QDs}

The $\mathrm{CdSe} / \mathrm{CdS} / \mathrm{ZnS}$ core/shell/shell QDs were synthesized as previously reported. ${ }^{[4]}$ Assynthesized QDs ligand were exchanged with $0.5 \mathrm{mmol}$ of LA and $0.5 \mathrm{mmol}$ of ZA were dissolved in $2 \mathrm{~mL}$ of deionized water. Then $2 \mathrm{mmol}$ of $\mathrm{NaBH}_{4}$ was added to the ligand solution, and it was stirred for $20 \mathrm{~min} .5 \mathrm{nmol}$ of QDs were dispersed in chloroform. The QD solution was added to ligand solution and stirred for $12 \mathrm{~h}$ at $45{ }^{\circ} \mathrm{C}$. The QDs migrated from the chloroform layer to the aqueous layer. The chloroform layer was discarded, then the aqueous suspension of QDs was dialyzed using an Amicon 50-kDa molecular weight cutoff (MWCO) centrifugal filter to remove excess ligand.

To increase colloidal stability, a second ligand exchange was conducted: 1 mmol of LA was dissolved in $2 \mathrm{~mL}$ of deionized water, then $2 \mathrm{mmol}_{\text {of }} \mathrm{NaBH}_{4}$ was added to the ligand solution, and it was stirred for $20 \mathrm{~min}$. Ligand-exchanged QDs in $2 \mathrm{~mL}$ of chloroform were added to the ligand solution, and the mixture was stirred for $12 \mathrm{~h}$ at $45^{\circ} \mathrm{C}$. The chloroform layer was removed, then the QD solution was dialyzed using the centrifugal filter.

$N$-(3-Dimethylaminopropyl)- $N$ '-ethylcarbodiimide hydrochloride (EDC, 100 equivalent to QDs) and $N$-Hydroxysulfosuccinimide sodium salt (sulfo-NHS, 200 equivalent to QDs) were added to ligand-exchanged QDs in $20 \mathrm{mM}$ pH 6.5 MES buffer, then the QD solution was vortexed for 15 min then dialyzed with MES buffer and 0.1 M pH 7.4 PBS buffer. Streptavidin in $0.1 \mathrm{M}$ PBS buffer (5 equivalent to QDs) was added to QDs solution and it was vortexed from 2 h,then dialyzed using a 100-kDa MWCO centrifugal filter.

The $0.2 \mathrm{nmol}$ of streptavidin conjugated QDs (QD-S) were added to $0.05 \mathrm{pmol}$ of SMNC-ZW$3+\mathrm{b}$ solution, and it was vortexed for $30 \mathrm{~min}$; the SMNC-ZW/Am were collected using a magnet, then excess SA-QDs were removed. Collected SMNC-ZW/Ams were re-dispersed in deionized water. 


\section{Fluorescein isothiocyanate (FITC) conjugation of bovine serum albumin (BSA)}

BSA $(0.066 \mathrm{~g})$ was dissolved in $10 \mathrm{~mL}$ of $\mathrm{NaHCO}_{3}$ buffer $(0.1 \mathrm{M}, \mathrm{pH} 9)$. Under continuous stirring, $38.6 \mu \mathrm{L}$ of FITC solution (in DMSO, $10 \mathrm{mg} / \mathrm{mL}$ ) was added to BSA solution, and it was stirred for $1 \mathrm{~h}$ in darkness. The solution was dialyzed using a 30-kDa MWCO centrifugal filter.

To quantify non-specific adsorption of protein, 10 nmol of FITC-BSA was mixed with $0.01 \mathrm{pmol}$ of SMNC-ZW/Am for $30 \mathrm{~min}$. Then SMNC-ZW/Ams were separated using a magnet, and excess FITC-BSA was discarded from the mixture. SMNC-ZW/Ams were redispersed in $2 \mathrm{~mL}$ of $\mathrm{NaHCO}_{3}$ buffer (0.01 M, pH 9).

\section{Amine quantification on SMNC-ZW by fluorescamine based assay}

For standard amine curve, various amount of APTES was dissolved at D.I. water (Analyte solution for standard curve). Similarly, 0.2 pmol of SMNC-ZW-2 and SMNC-ZW-3 were dispersed in $1 \mathrm{~mL}$ of D.I. water for quantification of surface amine. And then $0.1 \mathrm{~mL}$ of analyte solution and $0.6 \mathrm{~mL}$ of fluorescamine solution $(0.1 \mathrm{mg} / \mathrm{mL}$ in acetonitrile) were added to 1.3 $\mathrm{mL}$ of borate buffer (0.1 M, pH 8). $392 \mathrm{~nm}$ light was used as excitation source. Values of PL intensity at $478 \mathrm{~nm}$ were used for amine quantification of each nanoparticles.

\section{Salmonella bacteria separtaion}

For anti-Salmonella antibody conjugation, 0.4 pmol of SMNC-ZW-3 was dispersed at $2 \mathrm{~mL}$ PBS buffer solution. $0.2 \mathrm{~mL}$ of glutaraldehyde solution (5\% in D.I. water) was added to SMNCZW-3 solution and the solution was shaken for $2 \mathrm{~h}$. After 4 times washing with PBS buffer, SMNC-ZW-3 was dispersed at $2 \mathrm{~mL}$ PBS buffer solution. And then $0.04 \mathrm{~mL}$ of Salmonella antibody solution ( $2 \mathrm{mg} / \mathrm{mL}$ in PBS buffer) was added to glutaraldehyde conjugated SMNCZW-3 solution. After $2 \mathrm{~h}$ mixing, antibody conjugated SMNC-ZW-3 was separated using magnet and washed with PBS buffer solution. Washed nanoparticles were re-dispersed at D.I. 
water. $(0.2 \mathrm{pmol} / \mathrm{mL})$ For Salmonella separation experiment, $1 \mathrm{~mL}$ of Salmonella bacteria solution $\left(10^{8} \mathrm{CFU} / \mathrm{mL}\right)$ was spiked at $8.5 \mathrm{~mL}$ of milk. $0.5 \mathrm{~mL}$ of antibody conjugated SMNCZW-3 solution was added to Salmonella bacteria spiked milk and then mixed for $5 \mathrm{hr}$. SMNCZW-3 bounded bacteria was separated using magnet and washed with PBS buffer solution and D.I. water.

\section{Long term colloidal stability test in serum and saline}

To verification of long term stability, SMNC-ZW-2 and SMNC-ZW-3 was dispersed at $1 \mathrm{X}$ PBS buffer solution and fetal bovine serum solution (10\% FBS in 1X PBS buffer solution). To confirm colloidal stability, hydrodynamic size was measured for 7 days. 


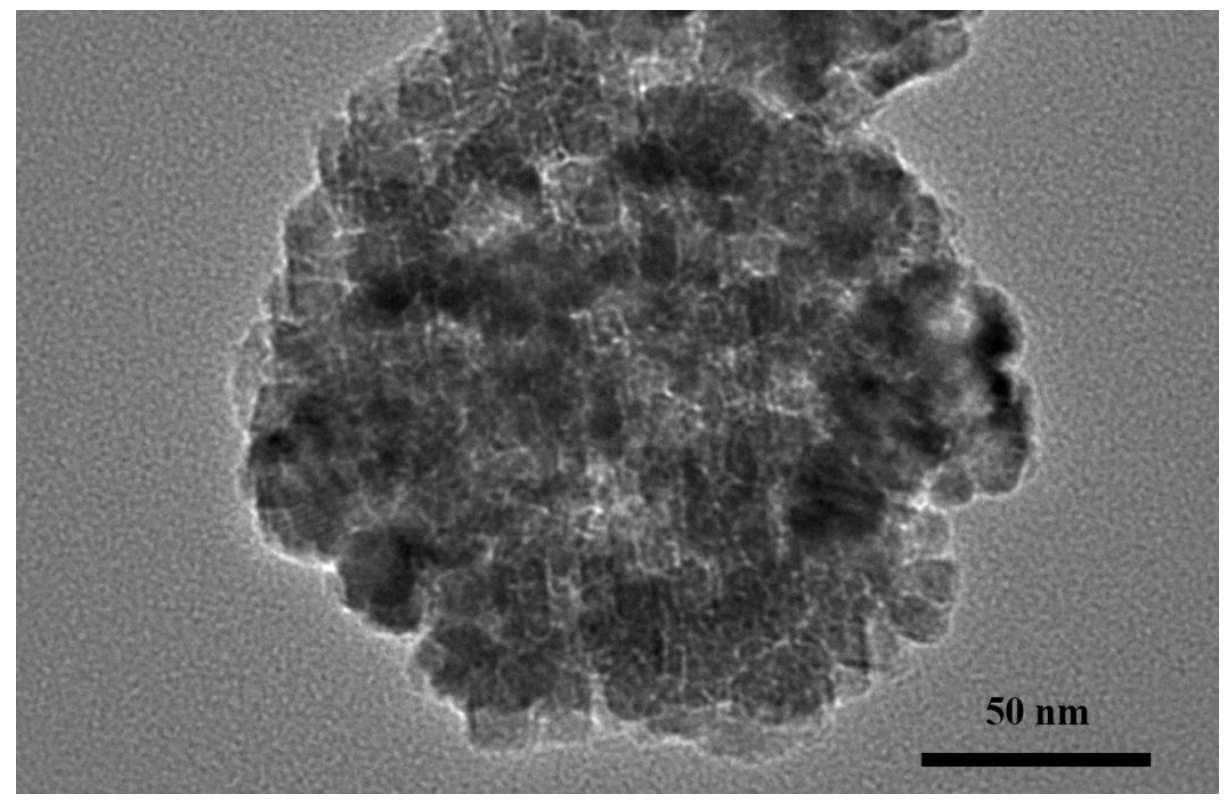

Figure S1. TEM image of single MNC. Each MNC was composed of small iron oxide nanoparticles of $15-20 \mathrm{~nm}$ size

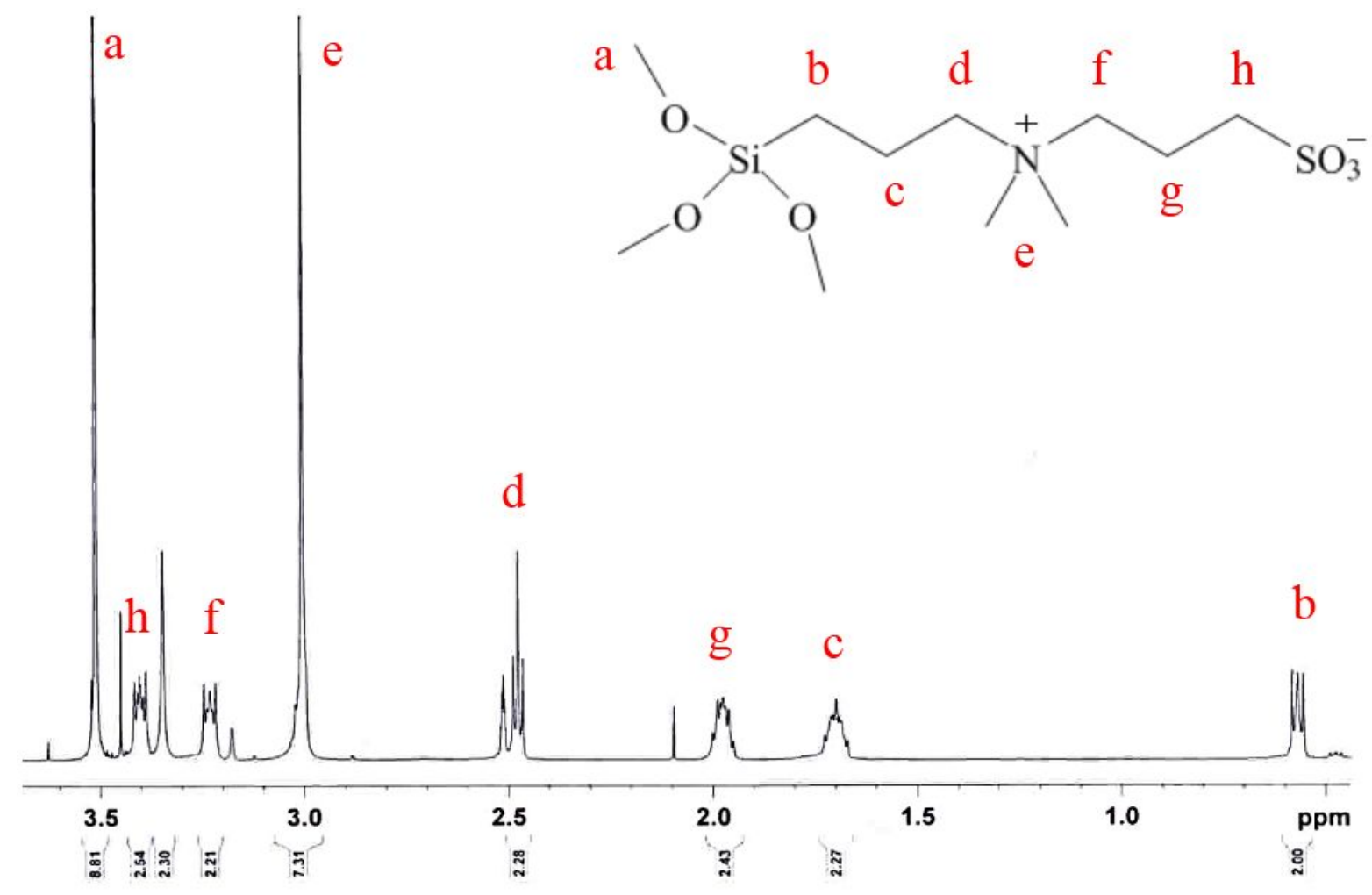

Figure S2. ${ }^{1} \mathrm{H}$ NMR spectrum of zwitterionic siloxane (ZW) dissolved in DMSO-d6 
a

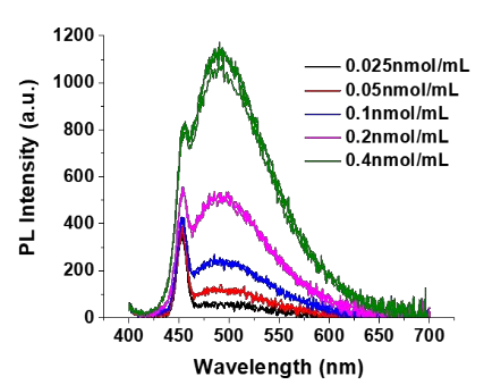

b

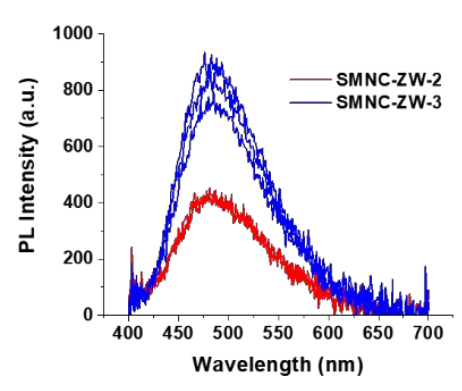

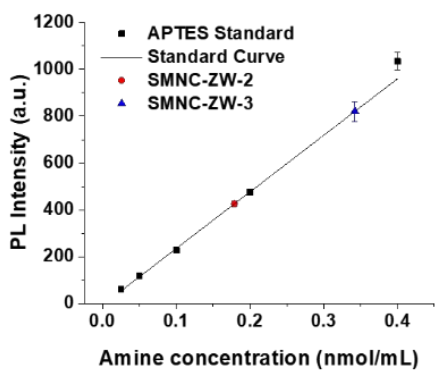

Figure S3. Fluorescamine assay for amine quantification on SMNC-ZW/Ams surface. PL spectrum of (a) standard APTES solution and (b) SMNC-ZW/Ams solution with fluorescamine. (c) PL intensity plotted as a function of amounts of amine group. 17891 ea and 34219 ea amine was existed on SMNC-ZW-2 and SMNC-ZW-3 surface.

a

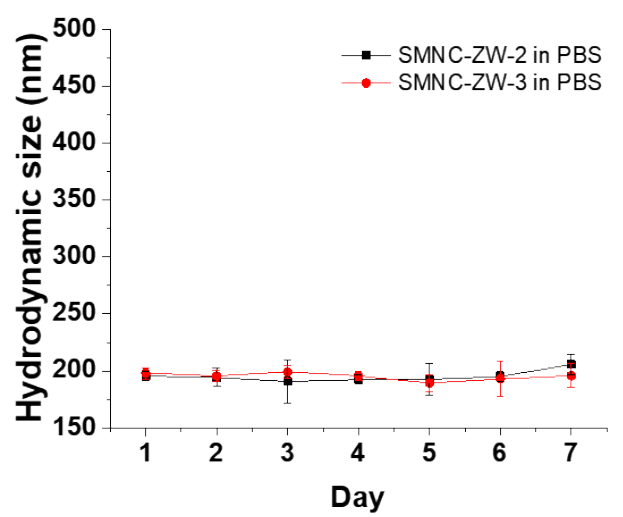

b

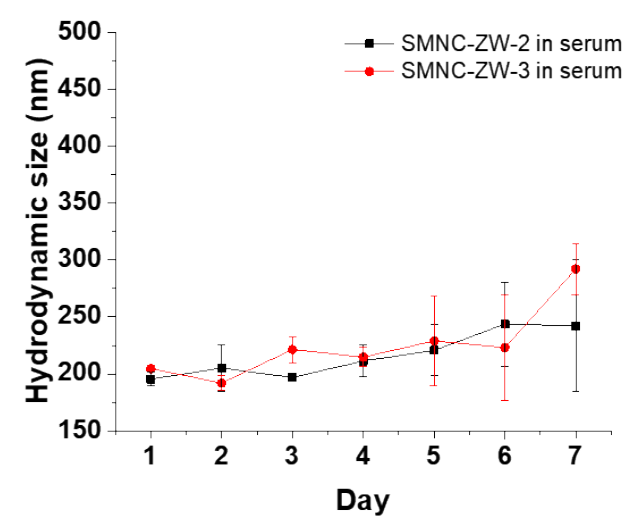

Figure S4. Hydrodynamic size of SMNC-ZW/Ams in 1X M PBS buffer solution (a) and fetal bovine serum solution (b).

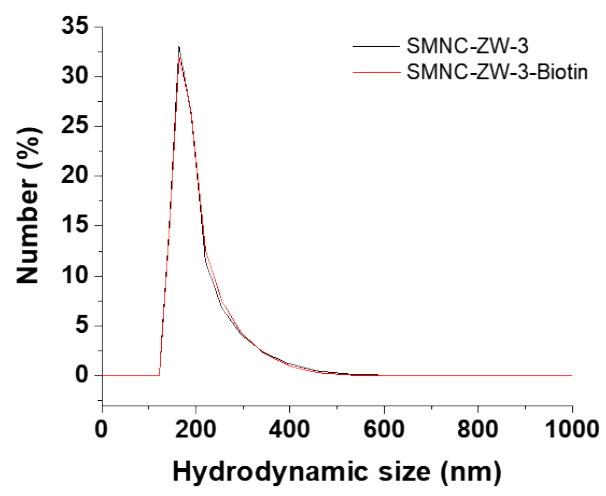

Figure S5. Hydrodynamic size of SMNC-ZW-3 (black line) and biotin conjugated SMNC-ZW3 (red line). 

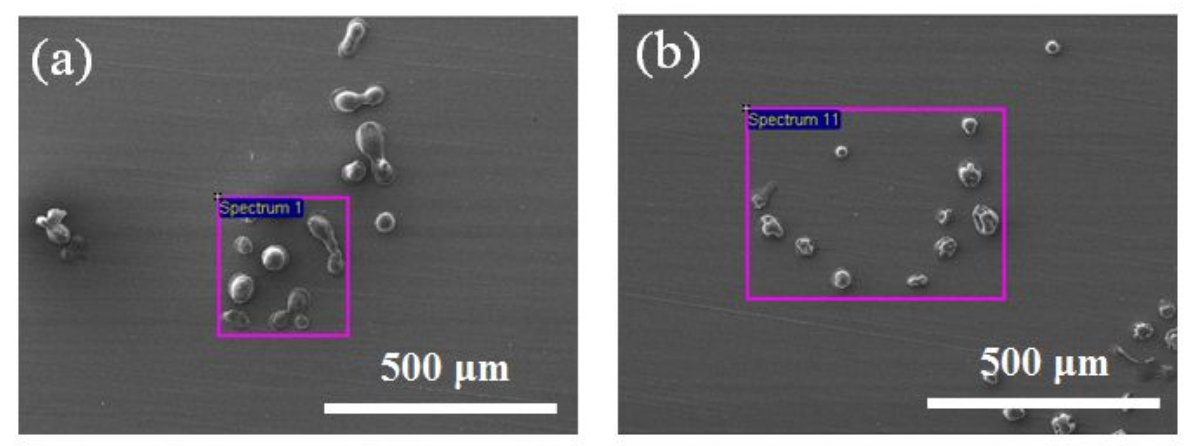

\begin{tabular}{c|cc|cc}
\multicolumn{1}{l|}{$(\mathrm{c})$} & \multicolumn{2}{|c|}{ Biotinylated SMNC-ZW/Am } & \multicolumn{2}{c}{ SMNC-ZW/Am } \\
\hline Element & Weight \% & Atomic \% & Weight \% & Atomic \% \\
\hline $\mathrm{C}$ & 91.38 & 98.01 & 100.00 & 100.00 \\
$\mathrm{Fe}$ & 8.62 & 1.99 & 0.00 & 0.00 \\
\hline Totals & 100.00 & 100.00 & 100.00 & 100.00
\end{tabular}

Figure S6. SEM images of (a) biotinylated SMNC-ZW/Am and (b) SMNC-ZW/Am. (c) Energy dispersive spectroscopy (EDS) analysis of streptavidin coated beads co-incubated with biotinylated SMNC-ZW/Am and SMNC-ZW/Am. The size of streptavidin coated beads was $45-165 \mu \mathrm{m}$.

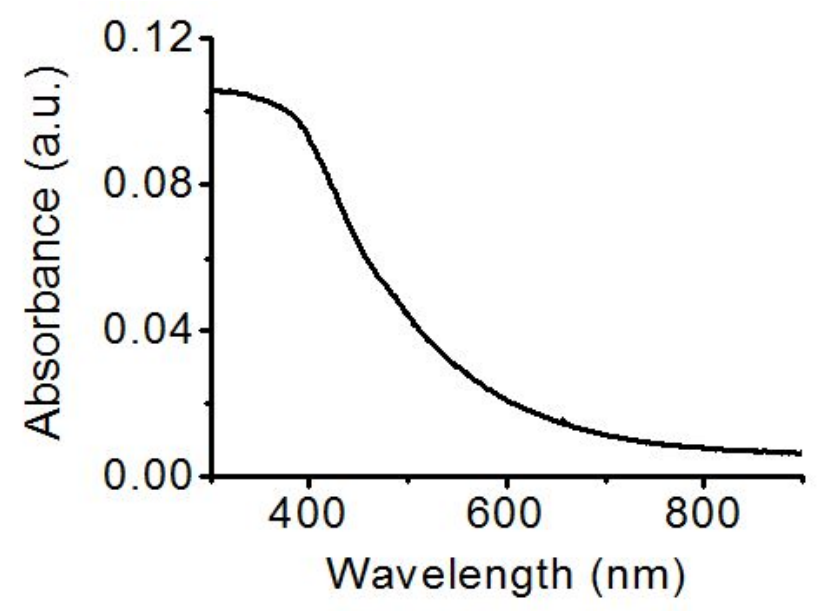

Figure S7. Absorption spectrum of SMNC. 


\section{Reference}

[1] W. Cheng, K. Tang, Y. Qi, J. Sheng and Z. Liu, J. Mater. Chem. 2010, 20, 1799.

[2] Z. G. Estephan, J. A. Jaber and J. B. Schlenoff, Langmuir, 2010, 26, 16884.

[3] J. Park, J. Nam, N. Won, H. Jin, S. Jung, S. Jung, S. H. Cho and S. Kim, Adv. Funct. Mater. 2011, 21, 1558.

[4] S. H. Bhang, N. Won, T. J. Lee, H. Jin, J. Nam, J. Park, H. Chung, H. S. Park, Y. E. Sung, S. K. Hahn, B. S. Kim and S. Kim, ACS Nano, 2009, 3, 1389. 